\title{
Quasi-Orthogonal Time Division Multiplexing and Its Performances in Rayleigh Fading Channels
}

\author{
Enchang Sun, ${ }^{1,2}$ Kechu Yi, ${ }^{2}$ Bin Tian, ${ }^{2}$ and Dongying Zhang ${ }^{3}$ \\ ${ }^{1}$ School of Electronic Information and Control Engineering, Beijing University of Technology, Beijing, China \\ ${ }^{2}$ State Key Laboratory of Integrated Services Networks (ISN), Xidian University, Xi'an, China
}

${ }^{3}$ Beijing Research Institute of ZTE Corporation, Beijing 100191, China

Correspondence should be addressed to Enchang Sun, ecsun@bjut.edu.cn

Received 10 January 2012; Accepted 26 January 2012

Academic Editors: A. Maaref and K. Teh

Copyright () 2012 Enchang Sun et al. This is an open access article distributed under the Creative Commons Attribution License, which permits unrestricted use, distribution, and reproduction in any medium, provided the original work is properly cited.

\begin{abstract}
This paper proposes an efficient transmission scheme, Quasi-Orthogonal Time Division Multiplexing (QOTDM), which employs the shift orthogonal property of the pulse function with raised-cosine spectral shape, and the signal waveforms are quasiorthogonal in time domain. Comparing to orthogonal frequency division multiplexing (OFDM), QOTDM is less sensitive to carrier frequency offset and power amplifier nonlinearities while keeping a similar spectral efficiency with OFDM due to singlecarrier characteristics. QOTDM is a suitable consideration for the downlink transmission such as in satellite communications. An upper bound of sample error probability (SER) is derived to evaluate the performance of QOTDM. Comparisons of QOTDM and OFDM in Rayleigh fading channels show that the proposed QOTDM system is better than that of OFDM system in terms of bit error rate (BER) in high $E_{b} / N_{o}$ regions.
\end{abstract}

\section{Introduction}

Orthogonal frequency-division multiplexing (OFDM) is a promising technique for high-speed data transmission in mobile communications $[1,2]$ due to its favorable properties such as high spectral efficiency, robustness to channel fading, and capability of handling multipath fading. However, there are many disadvantages in OFDM, for example, OFDM systems are very sensitive to carrier-frequency offsets (CFO) [3], since they can only tolerate offsets which are a fraction of the spacing between the subcarriers. That is, high accurate synchronization of the carrier frequency at the receiver is required, or there will be loss of orthogonality between the subcarriers. Moreover, in typical cases, the transmitted signals exhibit high peak-to-average power ratio (PAPR) [4], which means that an amplifier must either have a large linear operating range or it will lead to nonlinearities for the transmitted signals. For the inherent disadvantages of OFDM, on the one hand, many researchers try to present some effective schemes to overcome both CFO and PAPR issues of OFDM; on the other hand, single-carrier signal processing schemes have been investigated.
In this paper, a quasi-orthogonal time division multiplexing (QOTDM) system is proposed, which is a singlecarrier modulation technique and has high spectral efficiency while overcoming the CFO and PAPR drawbacks in OFDM. In Section 2, the basic principles of QOTDM system are investigated. In Section 3, an upper bound of sample error probability in QOTDM is derived. The performance comparisons of QOTDM and OFDM in Rayleigh fading channels are presented in Section 4. And conclusions are given in Section 5.

\section{Basic Principles of QOTDM}

2.1. Concept of QOTDM. It is well known that the function has shift orthogonal property, that is, for integer $k$, the following equation holds:

$$
\int_{-\infty}^{\infty} \frac{\sin \pi f}{\pi f} \cdot \frac{\sin \pi(f-k)}{\pi(f-k)} d f= \begin{cases}1, & k=0 \\ 0, & \text { else. }\end{cases}
$$

OFDM has high spectral efficiency by exploiting the shift orthogonal property shown in (1). 
In OFDM, this is achieved by making all the carriers orthogonal to each other, suppressing interference between the closely spaced carriers. Making the carriers for each channel orthogonal to one another allows them to be spaced very closely. Based on the basic principle of time-frequency duality for Fourier transform, it can be known that the function

$$
\sin c(t)=\frac{\sin (\pi t)}{(\pi t)}
$$

also has shift orthogonal property. That is, one can obtain an orthogonal time-division multiplexing (OTDM) system by exchanging the time variable $t$ and frequency variable $f$ in (1). Thus (2) has shift orthogonal property in the time domain instead of the shift orthogonal property in the frequency domain as in OFDM. In OTDM system, the waveform of each transmitted signal is composed of a number of overlapped Sinc functions with rectangular spectral shape. And the number is determined by the amount of the parallel substreams. However, the Sinc function has an infinite nonzero range and is impractical for implementation. Fortunately, a low-pass filter with raised-cosine spectral shape has an impulse response

$$
c(t)=\frac{\sin \pi t / T}{\pi t / T} \cdot \frac{\cos \alpha \pi t / T}{1-4 \alpha^{2} t^{2} / T^{2}},
$$

where $\alpha$ is the roll-off factor and $T$ denotes one-sided time duration of the main lobe of the shape pulse. $c(t)$ is similar to a Sinc function and has approximate orthogonality, that is, for all integers except $k=0$, it holds that

$$
d(k)=\int_{-\infty}^{+\infty} c(t) \cdot c(t-k T) d t \approx 0 .
$$

one calls this property quasi-orthogonality and $d(k)$ a quasiorthogonal function. If the quasi-orthogonal function family is employed in OTDM, then the quasi-orthogonal timedivision multiplexing (QOTDM) could be obtained.

2.2. QOTDM System Model. QOTDM is based on sampleinterweaving rather than bit-interweaving as in the conventional time-division multiplexing. On the one hand, each of the input sequences can be a sample sequence resulted from sampling any continuous signal, so that QOTDM can be applied to continuous wave time division multiplexing [5]. On the other hand, the multiple sample sequences (such as $N$ sample sequences) can also be obtained from sampling a complex envelope of digital modulation signal of a data stream. Therefore, QOTDM can also be applied to data transmission similar to OFDM. That is, a high bit rate stream is split up into $N$ parallel low bit rate-substreams, each substream is modulated and sampled into one sample sequence, respectively, and then the $N$ sample sequences are multiplexed into one sequence by means of sample interweaving. Finally, the multiplexed sample sequence is transformed into a continuous signal by pulse amplitude modulation (PAM) or quadrature amplitude modulation (QAM) for transmission via a continuous channel. And QOTDM can transmit multiple sample sequences $\left(X_{n}(m), n=1,2, \ldots, N\right.$, $m=\infty, \ldots,-1,0,1, \ldots, \infty)$ with time-division-multiplexing mode via a continuous channel. At first, the $N$ sample sequences are multiplexed into one by sample-interweaving, and then transformed into a continuous signal, by means of PAM or QAM. As long as the overall impulse response of the channel is equivalent to the quasi-orthogonal function and the sampling of received signals is entirely synchronous with the transmitted signal, the $N$ sample sequences can be completely separated with each other and can be exactly recovered at the receiver ignoring the difference of an amplifying factor. Thus multiple continuous signals can be transmitted in QOTDM at higher bandwidth efficiency with simple implementation by acting on the discrete samples of these signals.

The equivalent baseband QOTDM system is shown in Figure 1. At the transmitter, the $N$ input sample sequences and $N_{s}$ synchronous sequences are interwoven into one sequence, and then the sequence is modulated by PAM or QAM.

The transmitted signal $s(t)$ can be represented as

$$
s(t)=\sum_{i=0}^{N+N_{s}-1} d_{i, n} p(t-i T), \quad(n-1) T_{s} \leq t \leq n T_{s},
$$

where $T_{s}$ and $T$ are the sample interval and the PAM/QAM symbol duration, respectively, $d_{i, n}$ denotes the $n$th sample of the $i$ th sample sequence, and $p(t)$ is the sample shaping waveform.

A procedure of QOTDM is illustrated as follows.

(a) Suppose there are $N$ signals to be transmitted, and the bandwidth of each signal is not greater than $B \mathrm{~Hz}$. The $N$ signals are sampled in turns at a rate of $N F_{s}$ to get a sample sequence, where $F_{s}$ is a sampling rate, $B<F_{s}<2 B$. And each $N$ samples (i.e., one from each signal) are grouped together. Each sample in the sequence can be regarded as a symbol.

(b) $N_{s}$ synchronous and training symbols are placed equally among the $N$ samples/symbols. Thus, one gets $\left(N+N_{s}\right)$ samples in a group, called one frame.

(c) For each frame, $M$ zero sample (here, $M=1$ ) is inserted between every two adjacent samples to implement the operation of upsampling, shown in Figure 2.

(d) After the insertion of zero samples, the frame passes through a pulse shaping filter for transmission.

The synchronous sequences are specially designed in [5], which can be used not only as a synchronous signal but also as a training sequence for channel equalization. Each QOTDM frame consists of $\left(N+N_{s}\right)$ samples, and the multiplexed sample sequence is converted into a continuous signal by PAM or QAM. At the receiver, an adaptive channel equalizer is employed to make the impulse response of the overall (transmitter, channel, and receiver) equivalent channel satisfy the First Nyquist criterion. The channel equalizer is a finite impulse response (FIR) adaptive filter 


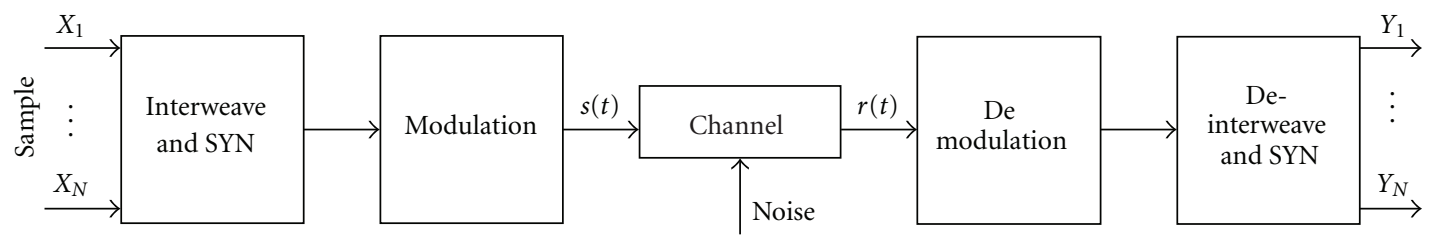

Figure 1: Equivalent baseband of QOTDM system.

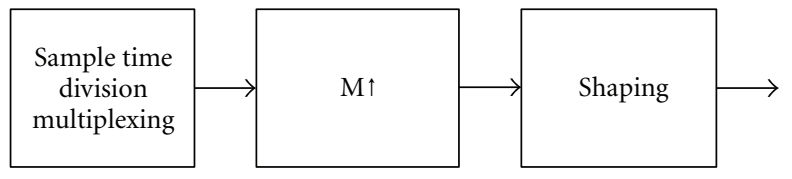

FIGURE 2: Interweaving block of QOTDM system.

of $\left(N+N_{s}\right)$ th order. If the received signal is sampled with the same sampling rate and at the accurate locations as the transmitter, then demultiplexed with the help of the synchronous sequence, one can recover the input parallel signals.

2.3. Bandwidth Comparison of QOTDM and OFDM. For a QOTDM system of $N$ subchannels, let the bit rate of the input bit-stream be $R_{b}=1=T_{b}$, split up into $N$ bit steams of bit-rate $R_{s}=1=N T_{b}$ for each, and then modulated into $N$ sample sequences, respectively, by using BPSK modulation with a raised cosine shaping FIR filter of a role-off factor $\alpha$.

The bandwidth of every signal should be $(1+\alpha) R_{s}$. After the $N$ sequences are multiplexed and added with $N_{s}$ synchronous sequences, the total bandwidth of QOTDM is

$$
\left(N+N_{s}\right)(1+\alpha) R_{s}=\frac{\left(N+N_{s}\right)(1+\alpha) R_{b}}{N} .
$$

The bandwidth efficiency of QOTDM with BPSK is

$$
\eta_{B}=\frac{N}{\left(\left(N+N_{s}\right)(1+\alpha)\right)}, \quad \text { bps } / \mathrm{Hz} .
$$

For an OFDM system of $N$ substreams with BPSK, the bandwidth efficiency is

$$
\eta_{\mathrm{BO}}=\frac{N}{\left(N+N_{\mathrm{cp}}\right)}, \quad \mathrm{bps} / \mathrm{Hz},
$$

where $N_{\text {cp }}$ denotes the length of the cyclic prefix of OFDM.

Generally speaking, the bandwidth efficiency of QOTDM is only a little lower than that of OFDM due to the role-off factor $\alpha$, if $N_{s}$ equals to $N_{\mathrm{cp}}$. However, QOTDM appears to be more robust against multipath fading [6]. Furthermore, QOTDM does not have a high peak-to-average power ratio as OFDM because only one carrier is used in QOTDM.

2.4. Applications of QOTDM. The concept and implementation of QOTDM is rather straightforward, and it finds applications in many scenarios, such as for the downlink in satellite communications system, and for wireless transmission of multichannel electronic waves of human body. That is, if multiple signals are amplified, transmitted simultaneously, one can significantly reduce the complexity and cost of the equipment by QOTDM.

\section{An Upper Bound of Sample Error Probability for QOTDM}

Let us consider the situation that the samples are quantized, each sample may take one of $D$ values, and it is assumed that $D$ is even. The allowed value of $D$ is given by

$$
a_{i}=2_{i}-D-1, \quad i=1,2, \ldots, D .
$$

The samples of a frame can be considered independent since they are sampled from $N$ parallel signals. Each sample takes one of $D$ values at random. If the intersample interference $I$ has $K$ nonzero terms, then $I$ has a discrete probability distribution consisting of $(D-1)^{K}$ allowed values with equal probability. If $I_{j}$ is an allowed value of $I$, then $-I_{j}$ is also an allowed value. The probability distribution of $I$ is, therefore, symmetric about zero. At the receiver, the received symbol is sampled periodically at the times $m T_{s}+\tau,(m=$ $0, \pm 1, \pm 2, \ldots) . \tau$ lies in $\left[0, T_{s}\right]$, and it would be chosen in a, manner, which optimizes the system performance.

The sampled signal takes the form

$$
\begin{aligned}
r\left(m T_{s}+\tau\right) & =\sum_{k=-\infty}^{\infty} d_{k} p\left(m T_{s}+\tau-k T_{s}\right)+n\left(m T_{s}+\tau\right) \\
& =d_{m} p(\tau)+\sum_{k \neq 0} d_{m-k} p\left(k T_{s}+\tau\right)+n\left(m T_{s}\right) \\
& =d_{m} p(\tau)+I+n .
\end{aligned}
$$

The first term in (10) is the desired signal, while the second and third terms are intersample interference and Gaussian noise, respectively, similar to that of conventional intersymbol interference, and $n$ is zero mean with variance $\sigma_{n}^{2}$.

For the sake of simplicity, we rewrite $I+n$ as $z$ and $|p(\tau)|$ as $p_{\tau}$. It is easily seen that the probability distribution of $z$ is symmetric about zero due to $n$ being zero means. Thus, when $a_{i}=-D+1$, an error results if $z>p_{\tau}$. If $a_{i}=D-1$, 
the condition for error is $z<p_{\tau}$. In all other cases, either condition results an error. The overall error probability is, therefore,

$$
P_{e}=\frac{2(M-1)}{M} P\left(z>p_{\tau}\right) .
$$

Since the terms of interference are finite and each quantized level is no larger than the largest quantified level, it can be considered that the total interference $I$ lies in $[-A,+A]$ and $A$ is some sufficient larger number, and let $f(x)$ denote the probability density function (pdf) of $I$. Since $I$ and $n$ are independent, the pdf of $z$ is in the form

$$
f(z)=\int_{-\infty}^{\infty} f(x) f(n=z-x) d x,
$$

where $f(n)=\left(2 \pi \sigma_{n}^{2}\right)^{-1 / 2} \exp \left(-n^{2} / 2 \sigma_{n}^{2}\right)$.

Considering that $I$ lies in $[-A,+A],(12)$ can be rewritten as

$$
f(z)=\frac{1}{\sigma_{n} \sqrt{2 \pi}} \int_{-A}^{A} f(x) \exp \left[-\frac{(z-x)^{2}}{2 \sigma_{n}^{2}}\right] d x .
$$

By using (13), we can rewrite (11) as

$$
\begin{aligned}
P_{e} & =\frac{2(M-1)}{M} P\left(z>p_{\tau}\right) \\
& =\frac{\sqrt{2}(M-1)}{\sigma_{n} M \sqrt{\pi}} \int_{-A}^{A} f(x) \int_{p_{\tau}-x}^{\infty} \exp \left[-\frac{z^{2}}{2 \sigma_{n}^{2}}\right] d z d x .
\end{aligned}
$$

Applying the modified Chernoff bound [7], we get

$$
\int_{p_{\tau}-x}^{\infty} \exp \left[-\frac{z^{2}}{2 \sigma_{n}^{2}}\right] d z \leq \frac{\exp \left[\left(s^{2} \sigma_{n}^{2} / 2\right)-s\left(p_{\tau}-x\right)\right]}{s},
$$$$
x>0 \text {. }
$$

Substituting (15) into (14) yields

$$
\begin{aligned}
P_{e} & =\frac{2(M-1)}{M} P\left(z>p_{\tau}\right) \\
& \leq \frac{\sqrt{2}(M-1) \exp \left[\left(s^{2} \sigma_{n}^{2} / 2\right)-s p_{\tau}\right]}{s \sigma_{n} M \sqrt{\pi}} \int_{-A}^{A} f(x) \exp [s x] d x .
\end{aligned}
$$

By setting the derivative of the right side of (16) with respect to $s$ to zero, we can find the value of $s$ which makes $P_{e}$ get its upper bound

$$
s=\frac{\left(p_{\tau}-x+\sqrt{\Delta}\right)}{\left(2 \sigma_{n}^{2}\right)},
$$

where $\Delta=\left(p_{\tau}-x\right)^{2}+4 \sigma_{n}^{2}$.

The main advantage of (16) is that it can be efficiently applied in the evaluation of the sample error performance of QOTDM in the presence of additive white Gaussian noise. In addition, (17) is helpful to get the upper bound in QOTDM system.

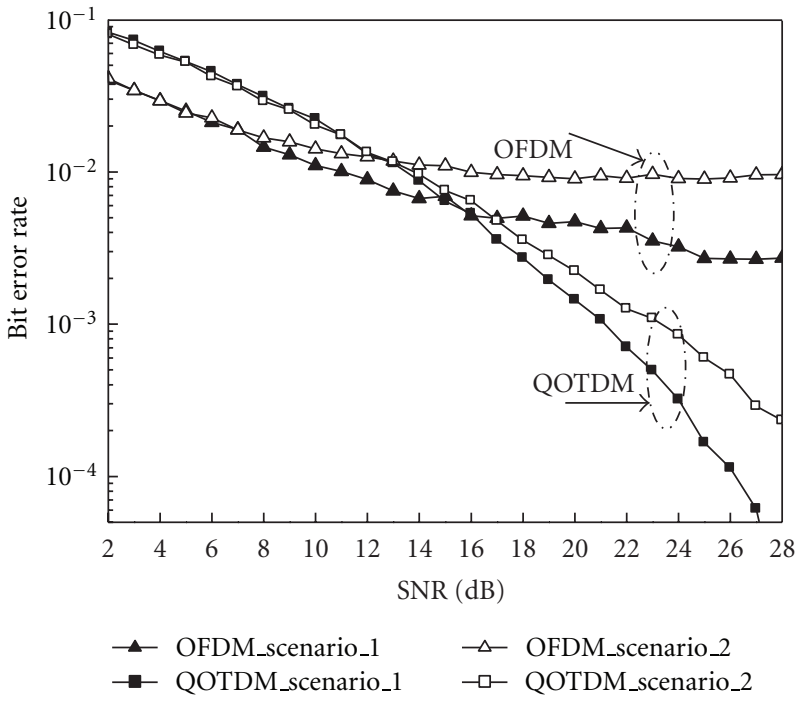

Figure 3: Bit Error Rate of QOTDM and OFDM system.

\section{Performance Comparison of QOTDM and OFDM in Rayleigh Fading Channels}

Computer simulations are performed to compare the performance of QOTDM system with that of OFDM system. In the simulation, $N=30$ parallel substreams and 2 synchronous sequence substreams were considered in QOTDM system and QAM modulation. A raised cosine pulse shaping with roll-off factor of 0.35 and band-limitation effects were also included. Due to 32 parallel sub-streams (including 30 data streams and 2 synchronous streams) considered in QOTDM system, the corresponding OFDM system should have $N=$ 32 subcarriers to make the simulation comparison fairly as possible. The length of Cyclic Prefix (CP) is 8, and QAM modulation is employed.

In the comparison, the same data symbols were considered in both QOTDM and OFDM systems. Two Rayleigh fading models named scenario- 1 and scenario- 2 are considered. In scenario-1, the channel varies independently during 40 samples duration, and in scenario-2, the channel varies during 10 samples duration.

From Figure 3, one can see that the bit error rate (BER) of OFDM has an error floor in the two scenarios mentioned above, while it does not exist in QOTDM system.

It also can be seen that the BER of QOTDM is lower than that of OFDM in high signal-to-noise ratio (SNR). This is mainly because in fast fading channels, the impulse response has varied during the inverse fast Fourier transform (IFFT) integral, and the fast Fourier transform (FFT) operation is applied to the fading signals, which have been varied differently due to the channel. After FFT operation at the receiver, the original signal (before IFFT) cannot be obtained properly due to the channel variation in one OFDM symbol duration time. While in QOTDM, the samples are interleaved before transmission, and at the receiver, an adaptive equalizer is employed to make the impulse response of the overall equivalent channel satisfy the First Nyquist 
criterion as possible. All these make the signal suffer much less from fading channel, which makes QOTDM have higher performance than OFDM.

\section{Conclusions}

We have presented an efficient transmission scheme, QuasiOrthogonal Time Division Multiplexing (QOTDM), which does not suffer the drawbacks, inherent in OFDM systems, that is, high sensitivity to carrier frequency offset (CFO) and high peak-to-average power ratio (PAPR), due to its single carrier modulation. And multiple continuous signals can be transmitted in QOTDM at higher band efficiency with simple implementation by acting on the discrete samples of these signals. An upper bound of SER is also derived to evaluate the performance of QOTDM systems.

From computer simulations, it is clear that the proposed QOTDM system is better than that of OFDM system in terms of bit error rate (BER) in the high $E_{b} / N_{o}$ regions. In the low $E_{b} / N_{o}$ region, the bit error rate of QOTDM is higher than that of OFDM. The reason is that, at low $E_{b} / N_{o}$, less information of the channel can be obtained and the channel equalization is not well enough. However, with the increasing of the $E_{b} / N_{o}$, the channel equalization can be better, so the QOTDM system can provide much better performance than OFDM.

\section{Acknowledgments}

Part of this paper has been presented at AINA'07. This work was sponsored by the State Key lab. of ISN, Xidian University (ISN10-02) and the Scientific Research Foundation for the Doctor degree, Beijing University of Technology (X0002012200903). It was also supported by the National Natural Science Foundation of China (Grant no. 61072088). The authors thank the referees for their helpful comments and contribution.

\section{References}

[1] J. A. C. Bingham, "Multicarrier modulation for data transmission: an idea whose time has come," IEEE Communications Magazine, vol. 28, no. 5, pp. 5-14, 1990.

[2] J. Perelló, S. Spadaro, J. Comellas, and G. Junyent, "A load-based reflection routing protocol with resource pre-allocation for contention resolution in OBS networks," European Transactions on Telecommunications, vol. 20, no. 1, pp. 1-7, 2009.

[3] P. H. Moose, "Technique for orthogonal frequency division multiplexing frequency offset correction," IEEE Transactions on Communications, vol. 42, no. 10, pp. 2908-2914, 1994.

[4] C. Tellambura, "Upper bound on peak factor of N-multiple carriers," Electronics Letters, vol. 33, no. 19, pp. 1608-1609, 1997.

[5] K.-C. Yi, C. Y. Gu, and C. T. Wang, "Continuous wave timedivision-multiplexing and its applications," IEICE Transactions on Communications, vol. E88-B, no. 11, pp. 4266-4273, 2005.

[6] E. Sun, B. Tian, Y. Wang, and K. Yi, "Quasi-orthogonal time division multiplexing and its applications under rayleigh fading channels," in Proceedings of the 21st International Conference on Advanced Information Networking and ApplicationsWorkshops/Symposia (AINAW'07), pp. 172-176, May 2007.

[7] J. J. O'Reilly and J. E. Mitchell, "Simplified derivation of the modified Chernoff upper bound," JIEE Proceedings, vol. 152, no. 6 , pp. $850-854$. 

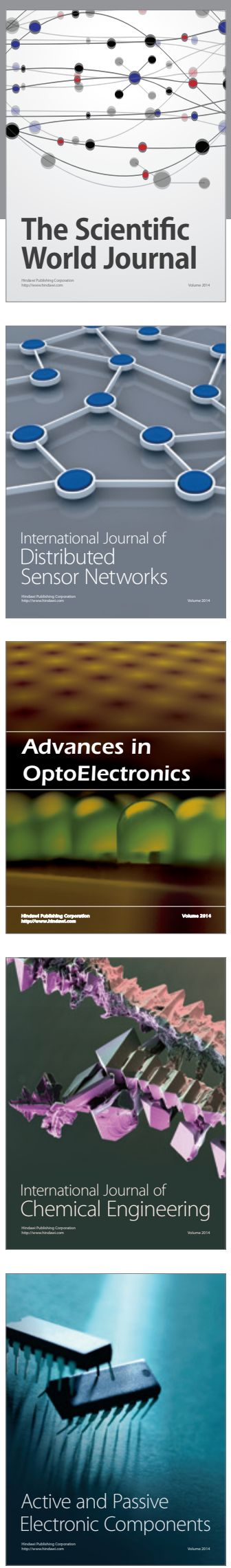
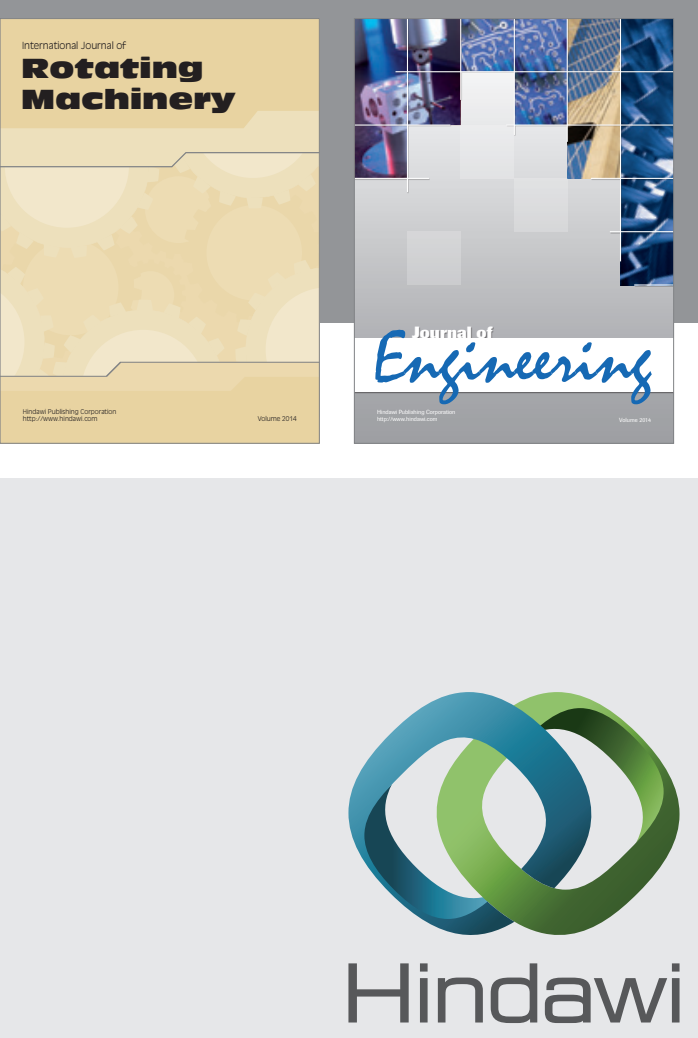

Submit your manuscripts at

http://www.hindawi.com
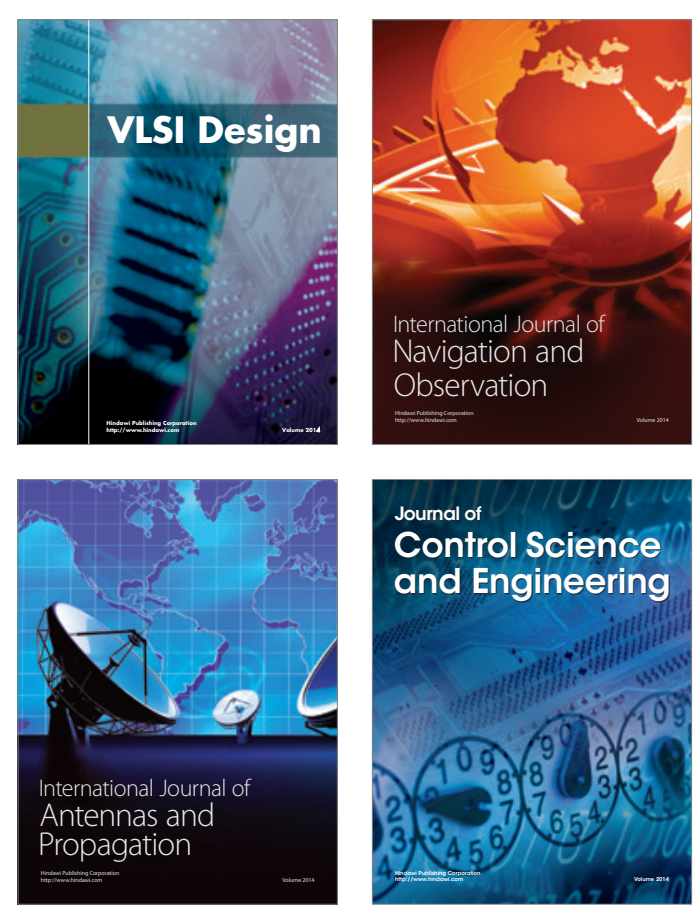
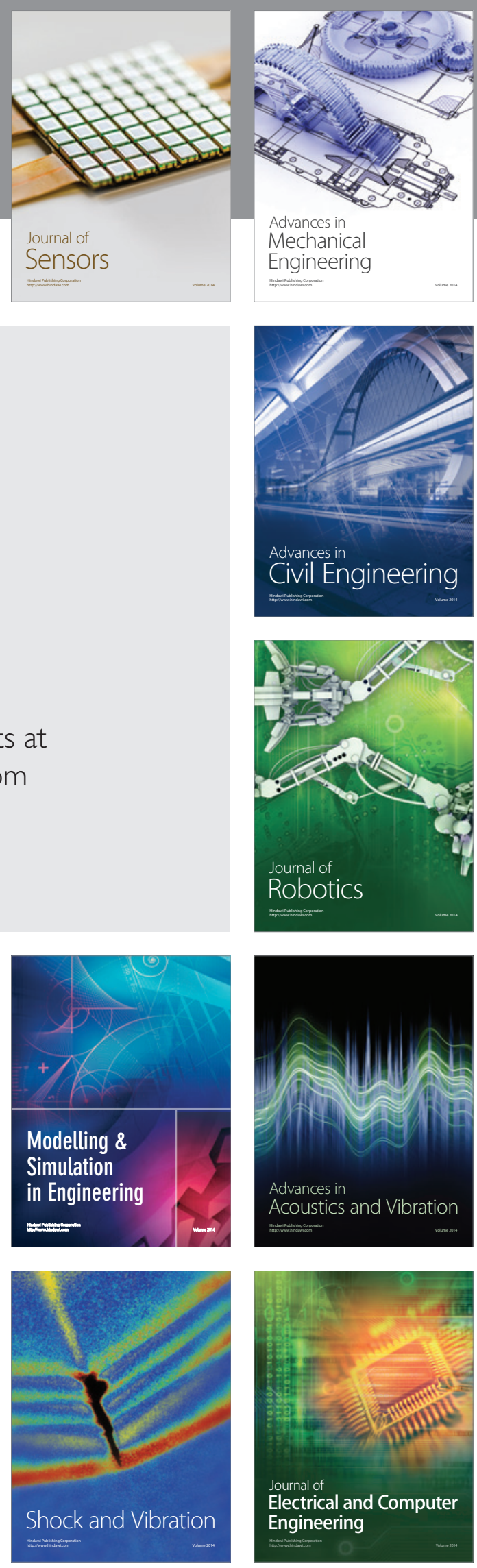